This is a postprint version of the following published document:

Bosuwan, N.; López Lagomasino, G. (2015). "Inverse Theorem on Row Sequences of Linear Padé-orthogonal Approximation". Computational Methods and Function Theory, 15, pp. 529-554.

DOI: $10.1007 / \mathrm{s} 40315-015-0121-3$

Proyecto MTM2012-36732-C03-01

(C) Springer 2015 


\title{
Inverse Theorem on Row Sequences of Linear Padé-orthogonal Approximation
}

\section{N. Bosuwan 1,2 - G. López Lagomasino 3}

\begin{abstract}
We give necessary and sufficient conditions for the convergence with geometric rate of the denominators of linear Padé-orthogonal approximants corresponding to a measure supported on a general compact set in the complex plane. Thereby, we obtain an analog of Gonchar's theorem on row sequences of Padé approximants.
\end{abstract}

Keywords Padé approximation · Padé-orthogonal approximation · Orthogonal polynomials · Fourier-Padé approximation · Inverse problems

Mathematics Subject Classification Primary 30E10 - 41A27; Secondary 41A21

Dedicated to Professor Edward B. Saff on the occasion of his 70th birthday.

Communicated by Doron Lubinsky.

The research of N. Bosuwan is supported by Mahidol University. The research of G. López Lagomasino is supported by Ministerio de Economía y Competitividad under Grant MTM2012-36732-C03-01.

N. Bosuwan

nattapong.bos@mahidol.ac.th

G. López Lagomasino

lago@math.uc3m.es

1 Department of Mathematics, Faculty of Science, Mahidol University, Rama VI Road, Ratchathewi District, Bangkok 10400, Thailand

2 Centre of Excellence in Mathematics, CHE, Si Ayutthaya Road, Bangkok 10400, Thailand

3 Departamento de Matemáticas, Universidad Carlos III de Madrid, c/Avda. de la Universidad, 30, 28911 Leganés, Spain 


\section{Introduction}

In this paper, $E$ denotes a compact subset of the complex plane $\mathbb{C}$ which contains infinitely many points such that $\overline{\mathbb{C}} \backslash E$ is simply connected. There exists a unique exterior conformal representation $\Phi$ from $\overline{\mathbb{C}} \backslash E$ onto $\overline{\mathbb{C}} \backslash\{w:|w| \leq 1\}$ satisfying $\Phi(\infty)=\infty$ and $\Phi^{\prime}(\infty)>0$. Thus,

$$
\Phi(z)=\operatorname{cap}(E)^{-1} z+\mathcal{O}(1), \quad z \rightarrow \infty .
$$

It is well known that the constant $\operatorname{cap}(E)$ coincides with the logarithmic capacity of the compact set $E$ (see [14, p. 313]). Furthermore, we assume that $E$ is such that the inverse function $\Psi=\Phi^{-1}$ can be extended continuously to $\overline{\mathbb{C}} \backslash\{w:|w|<1\}$ (the closure of a bounded Jordan region and a finite interval satisfy the above conditions).

Let $\mu$ be a finite positive Borel measure with infinite support $\operatorname{supp}(\mu)$ contained in $E$. We write $\mu \in \mathcal{M}(E)$ and define the associated inner product,

$$
\langle g, h\rangle_{\mu}:=\int g(\zeta) \overline{h(\zeta)} \mathrm{d} \mu(\zeta), \quad g, h \in L_{2}(\mu)
$$

Let

$$
p_{n}(z):=\kappa_{n} z^{n}+\cdots, \quad \kappa_{n}>0, \quad n=0,1, \ldots,
$$

be the orthonormal polynomial of degree $n$ with respect to $\mu$ having positive leading coefficient; that is, $\left\langle p_{n}, p_{m}\right\rangle_{\mu}=\delta_{n, m}$. Denote by $\mathcal{H}(E)$ the space of all functions holomorphic in some neighborhood of $E$.

Definition 1 Let $F \in \mathcal{H}(E), \mu \in \mathcal{M}(E)$, and a pair of non-negative integers $(n, m)$ be given. A rational function $[n / m]_{F}^{\mu}:=P_{n, m}^{\mu} / Q_{n, m}^{\mu}$ is called an $(n, m)$ (linear) Padéorthogonal approximant of $F$ with respect to $\mu$ if $P_{n, m}^{\mu}$ and $Q_{n, m}^{\mu}$ are polynomials satisfying

$$
\begin{aligned}
& \operatorname{deg}\left(P_{n, m}^{\mu}\right) \leq n, \quad \operatorname{deg}\left(Q_{n, m}^{\mu}\right) \leq m, \quad Q_{n, m}^{\mu} \not \equiv 0, \\
& \left\langle Q_{n, m}^{\mu} F-P_{n, m}^{\mu}, p_{j}\right\rangle_{\mu}=0, \quad \text { for } \quad j=0,1, \ldots, n+m .
\end{aligned}
$$

Since $Q_{n, m}^{\mu} \not \equiv 0$, we normalize it to have leading coefficient equal to 1 .

Obviously, given $Q_{n, m}^{\mu}$,

$$
P_{n, m}^{\mu}(z)=\sum_{j=0}^{n}\left\langle Q_{n, m}^{\mu} F, p_{j}\right\rangle p_{j}(z)
$$

is uniquely determined.

It is easy to see that if $E=\{z \in \mathbb{C}:|z| \leq 1\}$ and $\mathrm{d} \mu=\mathrm{d} \theta / 2 \pi$ on the unit circle $\{z \in \mathbb{C}:|z|=1\}$, then the linear Padé-orthogonal approximants are exactly the classical Padé approximants. The concept of linear Padé-orthogonal approximants was 
first introduced by Maehly [22] in 1960. In fact, he considered linear Padé-orthogonal approximants only for the case when $\mathrm{d} \mu=\mathrm{d} x / \sqrt{1-x^{2}}$ on $[-1,1]$. These rational functions are called Padé-Chebyshev approximants (see [1]) or sometimes crossmultiplied approximants (see [13]). Later, Cheney defined linear Padé-orthogonal approximants in a general setting ( $E$ is not just a finite interval) in his book [12]. The study of linear Padé-orthogonal approximants has mainly concentrated on the case when $\mu$ is supported on a finite interval (see, e.g., [7, 8, 17, 18,21,34,38]). Suetin [34] was the first to prove the convergence of row sequences of linear Padé-orthogonal approximants on $[-1,1]$ for a general class of measures for which the corresponding sequence of orthonormal polynomials has ratio asymptotic behavior. Moreover, he also proved an inverse result [35] for row sequences of linear Padé-orthogonal approximants on $[-1,1]$ under the assumption that the denominators of the approximants converge with geometric rate to a certain polynomial of degree $m$. For measures satisfying Szegó's condition, Buslaev [7,8] obtained inverse type results without the requirement that the denominators converge geometrically. Some problems on the convergence of diagonal sequences of linear Padé-orthogonal approximants on $[-1,1]$ were considered in $[17,18,21,38]$. Some papers which consider measures $\mu$ supported on the unit circle are $[3,4,7,8]$. Bosuwan et al. gave in [6] direct and inverse results for row sequences of linear Padé-orthogonal approximants corresponding to measures supported on a general compact $E$ as described above (which we will discuss in details below). Note that linear Padé-orthogonal approximants have also been called linear Padé approximants of orthogonal expansions [39], Fourier-Padé approximants $[3,4,9]$, and orthogonal Padé approximants [7,8].

We would like to point out that there is another related construction called nonlinear Padé approximants of orthogonal expansions (see [36]). Unlike the classical case, these linear and non-linear Padé approximants of orthogonal expansions lead, in general, to different rational functions (see an example in [36]). We will restrict our attention in this paper to linear Padé-orthogonal approximants, and in the rest of this paper, we will omit the word "linear" when we refer to them.

Let us introduce some notation. For any $\rho>1$, we denote by

$$
\Gamma_{\rho}:=\{z \in \mathbb{C}:|\Phi(z)|=\rho\}, \quad \text { and } \quad D_{\rho}:=E \cup\{z \in \mathbb{C}:|\Phi(z)|<\rho\}
$$

a level curve of index $\rho$ and a canonical domain of index $\rho$, respectively. We denote by $\rho_{0}(F)$ the index $\rho>1$ of the largest canonical domain $D_{\rho}$ to which $F$ can be extended as a holomorphic function, and by $\rho_{m}(F)$ the index $\rho$ of the largest canonical domain $D_{\rho}$ to which $F$ can be extended as a meromorphic function with at most $m$ poles (counting multiplicities).

Let $\mu \in \mathcal{M}(E)$ be such that

$$
\lim _{n \rightarrow \infty}\left|p_{n}(z)\right|^{1 / n}=|\Phi(z)|
$$

uniformly inside $\mathbb{C} \backslash E$. Here and in what follows, the phrase "uniformly inside a domain" means "uniformly on each compact subset of the domain". The Fourier coefficient of $F$ with respect to $p_{n}$ is given by 


$$
F_{n}:=\left\langle F, p_{n}\right\rangle_{\mu}=\int F(z) \overline{p_{n}(z)} \mathrm{d} \mu(z)
$$

As for Taylor series (see, for example, [30, Thm. 6.6.1]), it is easy to show that

$$
\rho_{0}(F)=\left(\varlimsup_{n \rightarrow \infty}\left|F_{n}\right|^{1 / n}\right)^{-1}
$$

Additionally, the series $\sum_{n=0}^{\infty} F_{n} p_{n}(z)$ converges to $F(z)$ uniformly inside $D_{\rho_{0}(F)}$ and diverges pointwise for all $z \in \mathbb{C} \backslash \overline{D_{\rho_{0}(F)}}$. Therefore, if (3) holds, then

$$
Q_{n, m}^{\mu}(z) F(z)-P_{n, m}^{\mu}(z)=\sum_{k=n+m+1}^{\infty}\left\langle Q_{n, m}^{\mu} F, p_{k}\right\rangle_{\mu} p_{k}(z)
$$

for all $z \in D_{\rho_{0}(F)}$.

We showed in [6, Ex. 1] that $[n / m]_{F}^{\mu}$ is not unique in general. However, if $\mu$ satisfies the condition

$$
\Delta_{n, m}(F, \mu):=\left|\begin{array}{cccc}
\left\langle F, p_{n+1}\right\rangle_{\mu} & \left\langle z F, p_{n+1}\right\rangle_{\mu} & \cdots & \left\langle z^{m-1} F, p_{n+1}\right\rangle_{\mu} \\
\vdots & \vdots & \vdots & \vdots \\
\left\langle F, p_{n+m}\right\rangle_{\mu} & \left\langle z F, p_{n+m}\right\rangle_{\mu} & \cdots & \left\langle z^{m-1} F, p_{n+m}\right\rangle_{\mu}
\end{array}\right| \neq 0
$$

or the condition that every solution of (1)-(2) has $\operatorname{deg} Q_{n, m}^{\mu}=m$, then $[n / m]_{F}^{\mu}$ is unique. Moreover, it is not difficult to verify that these two conditions are equivalent.

Let us introduce two classes of measures contained in $\mathcal{M}(E)$ which are relevant in what follows. We write $\mu \in \mathcal{R}(E)$ when the corresponding sequence of orthonormal polynomials has ratio asymptotics; that is,

$$
\lim _{n \rightarrow \infty} \frac{p_{n}(z)}{p_{n+1}(z)}=\frac{1}{\Phi(z)}
$$

We say that Szegő or strong asymptotics takes place, and write $\mu \in \mathcal{S}(E)$, if

$$
\lim _{n \rightarrow \infty} \frac{p_{n}(z)}{c_{n} \Phi^{n}(z)}=S(z) \quad \text { and } \quad \lim _{n \rightarrow \infty} \frac{c_{n}}{c_{n+1}}=1
$$

The first limit in (6) and the one in (5) are assumed to hold uniformly inside $\overline{\mathbb{C}} \backslash E$, the $c_{n}$ 's are positive constants, and $S(z)$ is a non-vanishing holomorphic function on $\overline{\mathbb{C}} \backslash E$. Clearly, (6) $\Rightarrow(5) \Rightarrow$ (3).

These two classes of measures have been well studied when the measure $\mu$ is supported on an interval of the real line or the whole unit circle (see, for example $[27,28])$ and characterized in terms of the analytic properties of the measure or of the corresponding sequences of recurrence coefficients (in case of the real line) or the Verblunsky coefficients (for the unit circle). For general compact sets $E$ contained in the complex plane the situation is not quite the same. There are many examples 
for which Szegő asymptotics takes place for measures supported on a single Jordan curve or arc (see [20,24,32,40-42]) and polynomials orthogonal with respect to area type measures on a Jordan region (see [11,23,25,31,33]). Outside the previously mentioned cases of the segment and the unit circle, the only case fully described and easily verifiable where $\mathcal{R}(E)$ is substantially larger than $\mathcal{S}(E)$ is when $E$ is an arc of the unit circle, see [2, Thm. 1] and [5, Thm. 1]. An interesting problem is to describe general measures in $\mathcal{R}(E)$ not necessarily in $\mathcal{S}(E)$, for different compact sets $E$.

In [6], direct and inverse results for row sequences of Padé-orthogonal approximants corresponding to a measure supported on a general compact set $E$ were proved. An analog of Montessus de Ballore's theorem (direct result) for Padé-orthogonal approximants is the following.

Theorem A Suppose $F \in \mathcal{H}(E)$ has poles of total multiplicity exactly $m$ in $D_{\rho_{m}(F)}$ at the (not necessarily distinct) points $\lambda_{1}, \ldots, \lambda_{m}$ and let $\mu \in \mathcal{R}(E)$. Then, $[n / m]_{F}^{\mu}$ is uniquely determined for all sufficiently large $n$ and the sequence $[n / m]_{F}^{\mu}$ converges

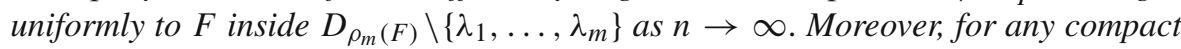
subset $K$ of $D_{\rho_{m}(F)} \backslash\left\{\lambda_{1}, \ldots, \lambda_{m}\right\}$,

$$
\varlimsup_{n \rightarrow \infty}\left\|F-[n / m]_{F}^{\mu}\right\|_{K}^{1 / n} \leq \frac{\max \{|\Phi(z)|: z \in K\}}{\rho_{m}(F)}
$$

where $\|\cdot\|_{K}$ denotes the sup-norm on $K$ and if $K \subset E$, then $\max \{|\Phi(z)|: z \in K\}$ is replaced by 1 . Additionally,

$$
\varlimsup_{n \rightarrow \infty}\left\|Q_{n, m}^{\mu}-Q_{m}\right\|^{1 / n} \leq \frac{\max _{1 \leq j \leq m}\left|\Phi\left(\lambda_{j}\right)\right|}{\rho_{m}(F)}<1
$$

where $\|\cdot\|$ denotes (for example) the norm induced in the space of polynomials of degree at most $m$ by the maximum of the absolute value of the coefficients and $Q_{m}(z)=\prod_{k=1}^{m}\left(z-\lambda_{k}\right)$.

In the same paper [6], an inverse type result in the spirit of Suetin's theorem in [37] was also obtained.

Theorem B Let $F \in \mathcal{H}(E), \mu \in \mathcal{S}(E)$, and $m$ be a fixed non-negative integer. If for all $n$ sufficiently large, $[n / m]_{F}^{\mu}$ has precisely $m$ finite poles $\lambda_{n, 1}, \ldots, \lambda_{n, m}$, and

$$
\lim _{n \rightarrow \infty} \lambda_{n, j}=\lambda_{j}, \quad j=1,2, \ldots, m
$$

$\left(\lambda_{1}, \ldots, \lambda_{m}\right.$ are not necessarily distinct $)$, then

(i) $F$ is holomorphic in $D_{\rho_{\min }}$ where $\rho_{\min }:=\min _{1 \leq j \leq m}\left|\Phi\left(\lambda_{j}\right)\right|$;

(ii) $\rho_{m-1}(F)=\max _{1 \leq j \leq m}\left|\Phi\left(\lambda_{j}\right)\right|$;

(iii) $\lambda_{1}, \ldots, \lambda_{m}$ are singularities of $F$; those lying in $D_{\rho_{m-1}(F)}$ are poles (counting multiplicities), and $F$ has no other poles in $D_{\rho_{m-1}(F)}$. 
It is still an open question whether Theorem B is valid under the weaker assumption $\mu \in R(E)$. However, we are not sure whether the result remains true with this degree of generality on the measure.

In this paper, we prove a reciprocal of Theorem A for row sequences of Padéorthogonal approximants (see Theorem 1 below). As compared with Theorem B, we must relax the condition on the measure to $\mu \in \mathcal{R}(E)$. To compensate, we will assume that the poles of the approximants converge with geometric rate as in (8). In contrast with Theorem B, we find that all the zeros of $Q_{m}$ are poles of $F$ and they all lie in $D_{\rho_{m}(F)}$. Combining Theorems 1 and A, we obtain Corollary 1 which characterizes the situation when $F$ has exactly $m$ poles in $D_{\rho_{m}(F)}$ (counting multiplicities) in terms of the exact rate of convergence in (8). This corollary is an analog of Gonchar's theorem for row sequences of classical Padé approximants (see, e.g., in [10, Sec. 1], [15, Sec. 3, Sec. 4] or [19, Sec. 2]).

An outline of the paper is as follows. In Sect. 2, we state the main theorem and its corollary. All auxiliary lemmas are in Sect. 3. Section 4 is devoted to the proof of the main result.

\section{Main Results}

The main theorem is the following.

Theorem 1 Let $F \in \mathcal{H}(E), \mu \in \mathcal{R}(E)$, and $m$ be a fixed non-negative integer. Assume that for all $n$ sufficiently large, $[n / m]_{F}^{\mu}$ has exactly $m$ finite poles and there exists a polynomial $Q_{m}(z)=\prod_{j=1}^{m}\left(z-\lambda_{j}\right)$ such that

$$
\varlimsup_{n \rightarrow \infty}\left\|Q_{n, m}^{\mu}-Q_{m}\right\|^{1 / n}=\delta<1 .
$$

Then

$$
\rho_{m}(F) \geq \frac{1}{\delta} \max _{1 \leq j \leq m}\left|\Phi\left(\lambda_{j}\right)\right|
$$

and in $D_{\rho_{m}(F)}$, the function $F$ has exactly $m$ poles at the points $\lambda_{1}, \ldots, \lambda_{m}$.

In [35, Thm. 1], Suetin proved this result for any measure $\mu$ supported on $[-1,1]$ such that $\mu^{\prime}>0$ almost everywhere on $[-1,1]$. Our proof of Theorem 1 is strongly influenced by the methods employed in that paper.

As a consequence of Theorems A and 1, we immediately have the following corollary.

Corollary 1 Let $F \in \mathcal{H}(E), \mu \in \mathcal{R}(E)$, and $m$ be a fixed non-negative integer. Then the following two assertions are equivalent:

(a) $F$ has exactly $m$ poles in $D_{\rho_{m}(F)}$.

(b) $[n / m]_{F}^{\mu}$ is uniquely determined and has precisely $m$ poles for all $n$ sufficiently large, and there exists a polynomial $Q_{m}$ of degree $m$ such that

$$
\varlimsup_{n \rightarrow \infty}\left\|Q_{n, m}^{\mu}-Q_{m}\right\|^{1 / n}=\delta<1 .
$$


Moreover, if either (a) or (b) holds, then the poles of $F$ in $D_{\rho_{m}(F)}$ coincide with the zeros $\lambda_{1}, \ldots, \lambda_{m}$ of $Q_{m}$ and

$$
\delta=\frac{\max _{1 \leq j \leq m}\left|\Phi\left(\lambda_{j}\right)\right|}{\rho_{m}(F)}
$$

It is natural to expect equality in (7) as a consequence of Theorem 1 . The idea is to show that a strict inequality in (7) leads to a better estimate in (8), which was shown to be exact, thus arriving at a contradiction. We have been unable to prove this in the context of Padé-orthogonal approximation though it holds in interpolation schemes of row sequences of Padé approximation.

\section{Auxiliary Lemmas}

The second type functions $s_{n}(z)$ defined by

$$
s_{n}(z):=\int \frac{\overline{p_{n}(\zeta)}}{z-\zeta} \mathrm{d} \mu(\zeta), \quad z \in \overline{\mathbb{C}} \backslash \operatorname{supp}(\mu)
$$

play a major role in our proof. The first lemma connects the asymptotic behavior of the orthonormal polynomials $p_{n}$ and that of the second type functions $s_{n}$.

Lemma 1 If $\mu \in \mathcal{R}(E)$, then

$$
\lim _{n \rightarrow \infty} p_{n}(z) s_{n}(z)=\frac{\Phi^{\prime}(z)}{\Phi(z)},
$$

uniformly inside $\overline{\mathbb{C}} \backslash E$. Consequently, for any compact set $K \subset \mathbb{C} \backslash E$, there exists $n_{0}$ such that $s_{n}(z) \neq 0$ for all $z \in K$ and $n \geq n_{0}$.

Proof of Lemma 1 See [6, Lem. 3.1]. In the proof of [6, Lem. 3.1], important use is made of the assumption $\mu \in \mathcal{R}(E)$ and the restrictions posed on the compact set $E$ (see also [26, Thm. 1.8]).

Recall that $\kappa_{n}$ is the leading coefficient of the orthonormal polynomial $p_{n}$. The second lemma shows that under the condition $\mu \in \mathcal{R}(E)$, the limit of the ratios of $\kappa_{n}$ is the capacity of $E$.

Lemma 2 If $\mu \in \mathcal{R}(E)$, then

$$
\lim _{n \rightarrow \infty} \frac{\kappa_{n}}{\kappa_{n+1}}=\operatorname{cap}(E),
$$

where cap $(E)$ is the capacity of $E$. 
Proof of Lemma 2 Since $\lim _{n \rightarrow \infty} z p_{n}(z) / p_{n+1}(z)=z / \Phi(z)$ holds uniformly inside $\overline{\mathbb{C}} \backslash E$, then

$$
\lim _{n \rightarrow \infty} \frac{\kappa_{n}}{\kappa_{n+1}}=\lim _{n \rightarrow \infty} \lim _{z \rightarrow \infty} \frac{z p_{n}(z)}{p_{n+1}(z)}=\lim _{z \rightarrow \infty} \lim _{n \rightarrow \infty} \frac{z p_{n}(z)}{p_{n+1}(z)}=\lim _{z \rightarrow \infty} \frac{z}{\Phi(z)}=\operatorname{cap}(E) .
$$

The next lemma is a curious relation of complex numbers which we will use at the end of the proof of Theorem 1.

Lemma 3 Let $N_{0} \in \mathbb{N}$ and $C>0$. If a sequence of complex numbers $\left\{F_{N}\right\}_{N \in \mathbb{N}}$ has the following properties:

(i) $\lim _{N \rightarrow \infty}\left|F_{N}\right|^{1 / N}=0$,

(ii) $\left|F_{N}\right| \leq C \sum_{k=N+1}^{\infty}\left|F_{k}\right|$, for all $N \geq N_{0}$,

then there exists $N_{1} \in \mathbb{N}$ such that $F_{N}=0$ for all $N \geq N_{1}$.

Proof of Lemma 3 Given the assumptions, there exists $M$ such that for all $N \geq M$,

$$
\left|F_{N}\right|^{1 / N}<\frac{1}{C+2}, \quad \text { and } \quad\left|F_{N}\right| \leq C \sum_{k=N+1}^{\infty}\left|F_{k}\right|
$$

We claim that for those $N$ 's,

$$
\left|F_{N}\right| \leq\left(\frac{C}{C+1}\right)^{n}\left(\frac{1}{C+2}\right)^{N}
$$

for any non-negative integer $n$. Then, letting $n \rightarrow \infty$, we see that $\left|F_{N}\right|=0$.

To prove the claim, we use induction on $n$. When $n=0$, the formula follows immediately from $\left|F_{N}\right|^{1 / N}<1 /(C+2)$. In general, using induction it follows that

$\left|F_{N}\right| \leq C \sum_{k=N+1}^{\infty}\left|F_{k}\right| \leq C \sum_{k=N+1}^{\infty}\left(\frac{C}{C+1}\right)^{n}\left(\frac{1}{C+2}\right)^{k}=\left(\frac{C}{C+1}\right)^{n+1}\left(\frac{1}{C+2}\right)^{N}$

This completes the proof.

\section{Proof of Theorem 1}

In the proof of Theorem 1, we mainly use the asymptotic properties of the orthogonal polynomials $p_{n}$ and the second type functions $s_{n}$ listed below.

From (5), it follows that

$$
\lim _{n \rightarrow \infty} \frac{p_{n}(z)}{p_{n+l}(z)}=\frac{1}{\Phi(z)^{l}}, \quad l=0,1, \ldots
$$


uniformly inside $\overline{\mathbb{C}} \backslash E$. By (11) and Lemma 1 for any $l, p=0,1, \ldots$, we have

$$
\lim _{n \rightarrow \infty} \frac{s_{n+l}(z)}{s_{n}(z)}=\lim _{n \rightarrow \infty} \frac{p_{n}(z)}{p_{n+l}(z)} \frac{p_{n+l}(z) s_{n+l}(z)}{p_{n}(z) s_{n}(z)}=\frac{1}{\Phi(z)^{l}} \frac{\Phi^{\prime}(z) / \Phi(z)}{\Phi^{\prime}(z) / \Phi(z)}=\frac{1}{\Phi(z)^{l}},
$$

uniformly inside $\overline{\mathbb{C}} \backslash E$. Furthermore,

$$
\lim _{n \rightarrow \infty}\left|p_{n}(z)\right|^{1 / n}=|\Phi(z)|
$$

and

$$
\lim _{n \rightarrow \infty}\left|s_{n}(z)\right|^{1 / n}=|\Phi(z)|^{-1}
$$

uniformly inside $\mathbb{C} \backslash E$, are trivial consequences of (11) and (12).

Proof of Theorem 1 We organize the proof of Theorem 1 as follows. First of all, we assume that in the region $D_{\rho_{m}(F)}$, the function $F$ has $k<m$ poles in $D_{\rho_{m}(F)}$ at the points $\tilde{\lambda}_{1}, \ldots, \tilde{\lambda}_{k}$. Set

$$
Q_{m}(z)=\prod_{j=1}^{m}\left(z-\lambda_{j}\right), \quad \tilde{Q}_{k}(z):=\prod_{j=1}^{k}\left(z-\tilde{\lambda}_{j}\right)=\prod_{w=1}^{\gamma}\left(z-\tilde{\alpha}_{w}\right)^{k_{w}},
$$

where $\tilde{\alpha}_{1}, \ldots, \tilde{\alpha}_{k}$ are distinct and $\sum_{w=1}^{\gamma} k_{w}=k$. Arguing as in the proof of $[6$, Thm. 2.1], our assumptions imply that the sequence $[n / m]_{F}^{\mu}(z)$ converges in capacity to $F$ inside $D_{\rho_{m}(F)}$, as $n \rightarrow \infty$. More precisely, for any $\varepsilon>0$ and any compact subset $K \subset D_{\rho_{m}(F)}$

$$
\lim _{n \rightarrow \infty} \operatorname{cap}\left(\left\{z \in K:\left|F(z)-[n / m]_{F}^{\mu}(z)\right| \geq \varepsilon\right\}\right)=0
$$

By Gonchar's lemma (see [16, Lem. 1] on page 507 and sentence at the beginning of that page regarding Cartan's inequality as well as the translator's correction), this implies that each $\tilde{\alpha}_{w}$ attracts at least $k_{w}$ poles of $[n / m]_{F}^{\mu}$ as $n \rightarrow \infty$. From this and (9), it follows that $\tilde{\lambda}_{1}, \ldots, \tilde{\lambda}_{k}$ are zeros of $Q_{m}$ and $Q_{m} F$ is holomorphic in $D_{\rho_{m}(F)}$. We can reindex $\lambda_{j}, \tilde{\lambda}_{j}, \tilde{\alpha}_{w}$ so that

$$
\begin{aligned}
& \lambda_{j}=\tilde{\lambda}_{j}, \quad j=1, \ldots, k, \quad \text { and } \quad \sigma_{w}:=\left|\Phi\left(\tilde{\alpha}_{w}\right)\right|, \quad w=1, \ldots, \gamma \\
& \left|\Phi\left(\lambda_{1}\right)\right| \leq \cdots \leq\left|\Phi\left(\lambda_{k}\right)\right|, \quad \text { and } \quad 1 \leq \sigma_{1} \leq \ldots \leq \sigma_{\gamma} .
\end{aligned}
$$

Next, we will prove by contradiction that the assumption $k<m$ on the number of poles of $F$ in $D_{\rho_{m}(F)}$ implies that $D_{\rho_{m}(F)}=\mathbb{C}$, i.e., $\rho_{m}(F)=\infty$. To this end, we show that if $\rho_{m}(F)<\infty$ then $F$ has at most $m$ poles in a canonical region which is strictly larger than $D_{\rho_{m}(F)}$ which clearly contradicts the definition of $\rho_{m}(F)$. This step is the main part of the proof of Theorem 1. Finally, we show that if $\rho_{m}(F)=\infty$ 
and $k<m$ then $F$ is a rational function with less than $m$ poles which contradicts the assumption that for all $n$ sufficiently large all the polynomials $Q_{n, m}^{\mu}$ have degree $m$. Thus, $F$ must have exactly $m$ poles in $D_{\rho_{m}(F)}$ and using Theorem A it follows that they must be the points $\lambda_{1}, \ldots, \lambda_{m}$ counting multiplicities.

Let us suppose that $F$ has $k<m$ poles at the points $\tilde{\lambda}_{1}, \ldots, \tilde{\lambda}_{k}$ in $D_{\rho_{m}(F)}$. The indices are taken so that $\tilde{\lambda}_{j}=\lambda_{j}, j=1, \ldots, k$. Let us prove that $D_{\rho_{m}(F)}=\mathbb{C}$. To the contrary, assume that $\rho_{m}(F)<\infty$. We plan to show that

$$
\varlimsup_{n \rightarrow \infty}\left|\left[F Q_{m}\right]_{n}\right|^{1 / n} \leq \frac{\delta}{\rho_{m}(F)}
$$

(Recall that $\left[F Q_{m}\right]_{n}:=\left\langle F Q_{m}, p_{n}\right\rangle_{\mu}$.) Combining this and (13), it follows that $Q_{m} F$ is holomorphic in $D_{\sigma}$, where $\sigma=\rho_{m}(F) / \delta>\rho_{m}(F)$. This implies that $F$ is meromorphic with at most $m$ poles in $D_{\sigma}$ which contradicts the definition of $\rho_{m}(F)$.

Now, let us prove (15). By the definition of Padé-orthogonal approximants, we have

$F(z) Q_{m}(z)=F(z)\left(Q_{m}(z)-Q_{n, m}^{\mu}(z)\right)+P_{n, m}^{\mu}(z)+\sum_{\nu=n+m+1}^{\infty}\left\langle Q_{n, m}^{\mu} F, p_{\nu}\right\rangle_{\mu} p_{v}(z)$,

which implies

$$
\left[F Q_{m}\right]_{n+b}=\left[F\left(Q_{m}-Q_{n, m}^{\mu}\right)\right]_{n+b}, \quad b=1, \ldots, m
$$

Applying Cauchy's residue theorem to the function $F\left(Q_{m}-Q_{n, m}^{\mu}\right)$ on the closed region $\bar{D}_{\rho} \backslash D_{r}:=\{z \in \mathbb{C}: r \leq|\Phi(z)| \leq \rho\}, 1<r<\sigma_{1}, \sigma_{\gamma}<\rho<\rho_{m}$, we obtain

$$
\begin{aligned}
{\left[F\left(Q_{m}-Q_{n, m}^{\mu}\right)\right]_{n+b} } & =\frac{1}{2 \pi i} \int_{\Gamma_{r}} F(t)\left(Q_{m}(t)-Q_{n, m}^{\mu}(t)\right) s_{n+b}(t) \mathrm{d} t \\
& =\frac{1}{2 \pi i} \int_{\Gamma_{\rho}} F(t)\left(Q_{m}(t)-Q_{n, m}^{\mu}(t)\right) s_{n+b}(t) \mathrm{d} t-\sum_{w=1}^{\gamma} \operatorname{res}\left(F\left(Q_{m}-Q_{n, m}^{\mu}\right) s_{n+b}, \tilde{\alpha}_{w}\right) \\
& =\frac{1}{2 \pi i} \int_{\Gamma_{\rho}} F(t)\left(Q_{m}(t)-Q_{n, m}^{\mu}(t)\right) s_{n+b}(t) \mathrm{d} t+\sum_{w=1}^{\gamma} \operatorname{res}\left(F Q_{n, m}^{\mu} s_{n+b}, \tilde{\alpha}_{w}\right) .
\end{aligned}
$$

Note that $F Q_{n, m}^{\mu} s_{n+b}$ is meromorphic on $\bar{D}_{\rho} \backslash D_{r}$ and has a pole at $\tilde{\alpha}_{w}$ of multiplicity at most $k_{w}$ for each $w=1, \ldots, \gamma$. Using the limit formula for residue, we have

$$
\operatorname{res}\left(F Q_{n, m}^{\mu} s_{n+b}, \tilde{\alpha}_{w}\right)=\frac{1}{\left(k_{w}-1\right) !} \lim _{z \rightarrow \tilde{\alpha}_{w}}\left(\left(z-\tilde{\alpha}_{w}\right)^{k_{w}} F(z) Q_{n, m}^{\mu}(z) s_{n+b}(z)\right)^{\left(k_{w}-1\right)}
$$


By the Leibniz formula and the fact that for $n$ sufficiently large, $s_{n}(z) \neq 0$ for $z \in \mathbb{C} \backslash E$ (see Lemma 1), we can transform the expression under the limit sign as follows

$$
\begin{aligned}
& \left(\left(z-\tilde{\alpha}_{w}\right)^{k_{w}} F(z) Q_{n, m}^{\mu}(z) s_{n+b}(z)\right)^{\left(k_{w}-1\right)} \\
& \quad=\left(\left(z-\tilde{\alpha}_{w}\right)^{k_{w}} F(z) Q_{n, m}^{\mu}(z) s_{n}(z) \frac{s_{n+b}(z)}{s_{n}(z)}\right)^{\left(k_{w}-1\right)} \\
& \quad=\sum_{p=0}^{k_{w}-1}\left(\begin{array}{c}
k_{w}-1 \\
p
\end{array}\right)\left(\left(z-\tilde{\alpha}_{w}\right)^{k_{w}} Q_{n, m}^{\mu}(z) F(z) s_{n}(z)\right)^{\left(k_{w}-1-p\right)}\left(\frac{s_{n+b}}{s_{n}}\right)^{(p)}
\end{aligned}
$$

To avoid long expressions, let us introduce the following notation

$$
\beta_{n}(w, p):=\frac{1}{\left(k_{w}-1\right) !}\left(\begin{array}{c}
k_{w}-1 \\
p
\end{array}\right) \lim _{z \rightarrow \tilde{\alpha}_{w}}\left(\left(z-\tilde{\alpha}_{w}\right)^{k_{w}} Q_{n, m}^{\mu}(z) F(z) s_{n}(z)\right)^{\left(k_{w}-1-p\right)}
$$

for $w=1, \ldots, \gamma$ and $p=0, \ldots, k_{w}-1$ and

$$
\eta(\rho, n, b):=\frac{1}{2 \pi i} \int_{\Gamma_{\rho}} F(t)\left(Q_{m}(t)-Q_{n, m}^{\mu}(t)\right) s_{n+b}(t) \mathrm{d} t, \quad b=1, \ldots, m
$$

(notice that the $\beta_{n}(w, p)$ do not depend on $b$ ). So, by (16) we can rewrite (17) as

$$
\left[F Q_{m}\right]_{n+b}=\eta(\rho, n, b)+\sum_{w=1}^{\gamma} \sum_{p=0}^{k_{w}-1} \beta_{n}(w, p)\left(\frac{s_{n+b}}{s_{n}}\right)^{(p)}\left(\tilde{\alpha}_{w}\right), \quad b=1, \ldots, m
$$

Since $k \leq m-1$, we have

$$
\sum_{w=1}^{\gamma} \sum_{p=0}^{k_{w}-1} \beta_{n}(w, p)\left(\frac{s_{n+b}}{s_{n}}\right)^{(p)}\left(\tilde{\alpha}_{w}\right)=\left[F Q_{m}\right]_{n+b}-\eta(\rho, n, b), \quad b=1, \ldots, k
$$

We will view (21) as a system of $k$ equations on the $k$ unknowns $\beta_{n}(w, p)$. If we can show that

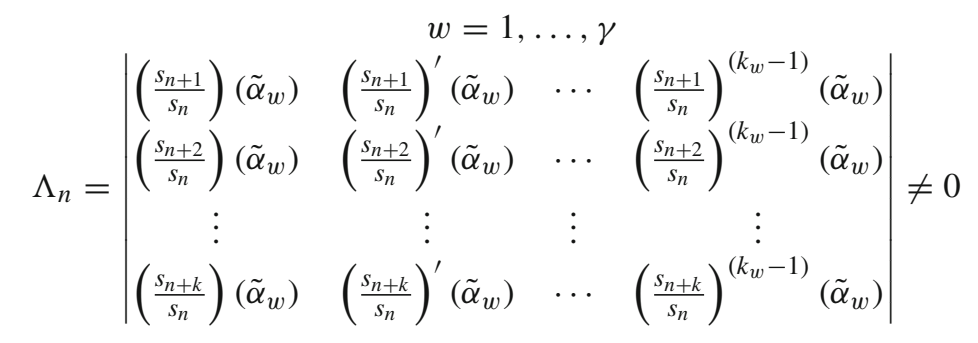

(this expression represents the determinant of order $k$ in which the indicated group of columns are successively written out for $w=1, \ldots, \gamma)$, then we can express $\beta_{n}(w, p)$ 
in terms of $\left(s_{n+b} / s_{n}\right)^{(p)}\left(\tilde{\alpha}_{w}\right)$ and $\left[F Q_{m}\right]_{n+b}-\eta(\rho, n, b)$. However, from (12) and the Weierstrass theorem, it follows that

$$
\begin{aligned}
\lim _{n \rightarrow \infty} \Lambda_{n}=\Lambda:=\left|\begin{array}{cccc}
R\left(\tilde{\alpha}_{w}\right) & R^{\prime}\left(\tilde{\alpha}_{w}\right) & \cdots & R^{\left(k_{w}-1\right)}\left(\tilde{\alpha}_{w}\right) \\
R^{2}\left(\tilde{\alpha}_{w}\right) & \left(R^{2}\right)^{\prime}\left(\tilde{\alpha}_{w}\right) & \cdots & \left(R^{2}\right)^{\left(k_{w}-1\right)}\left(\tilde{\alpha}_{w}\right) \\
\vdots & \vdots & \vdots & \vdots \\
R^{k}\left(\tilde{\alpha}_{w}\right) & \left(R^{k}\right)^{\prime}\left(\tilde{\alpha}_{w}\right) & \cdots & \left(R^{k}\right)^{\left(k_{w}-1\right)}\left(\tilde{\alpha}_{w}\right)
\end{array}\right| \\
=\prod_{w=1}^{\gamma}\left(k_{w}-1\right) ! ! \prod_{w=1}^{\gamma}\left(-\Phi^{\prime}\left(\tilde{\alpha}_{w}\right)\right)^{k_{w}\left(k_{w}-1\right) / 2} \prod_{w=1}^{\gamma} \\
\quad \times \Phi\left(\tilde{\alpha}_{w}\right)^{-k_{w}^{2}} \prod_{1 \leq i<j \leq \gamma}\left(\frac{1}{\Phi\left(\tilde{\alpha}_{j}\right)}-\frac{1}{\Phi\left(\tilde{\alpha}_{i}\right)}\right)^{k_{i} k_{j}} \neq 0,
\end{aligned}
$$

where $R(z)=1 / \Phi(z)$ and $n ! !=0 ! 1 ! \cdots \mathrm{n}$ ! (using for example [29, Thm. 1] for proving the last equality), for sufficiently large $n, \Lambda_{n} \neq 0$. Therefore, for all sufficiently large $n,\left|\Lambda_{n}\right| \geq c_{1}>0$ and we will only consider such $n$ below. Hereafter, $c_{1}, c_{2}, c_{3}, \ldots$ denote absolute constants which do not depend on $n$.

Applying Cramer's rule to (21), we have

$$
\begin{aligned}
\beta_{n}(w, p)=\frac{\Lambda_{n}(w, p)}{\Lambda_{n}}= & \frac{1}{\Lambda_{n}} \sum_{j=1}^{k}(-1)^{j+q(w, p)}\left(\left[F Q_{m}\right]_{n+j}\right. \\
& -\eta(\rho, n, j)) M_{n}(j, q(w, p))
\end{aligned}
$$

where $\Lambda_{n}(w, p)$ is the determinant obtained from $\Lambda_{n}$ replacing the column with index $q(w, p):=\left(\sum_{l=0}^{w-1} k_{l}\right)+p+1$ (where we define $\left.k_{0}:=0\right)$ with the column

$$
\left[\left(\left[F Q_{m}\right]_{n+1}-\eta(\rho, n, 1)\right) \quad \cdots \quad\left(\left[F Q_{m}\right]_{n+k}-\eta(\rho, n, k)\right)\right]^{T}
$$

and $M_{n}(j, q)$ is the $(j, q)$ th minor of $\Lambda_{n}(w, p)$. Substituting $\beta_{n}(w, p)$ in the formula (20) with the expression in (24) for $b=k+1$, we obtain

$$
\begin{aligned}
& {\left[F Q_{m}\right]_{n+k+1}=\eta(\rho, n, k+1)} \\
& \quad+\frac{1}{\Lambda_{n}} \sum_{w=1}^{\gamma} \sum_{p=0}^{k_{w}-1} \sum_{j=1}^{k}(-1)^{j+q(w, p)}\left(\left[F Q_{m}\right]_{n+j}-\eta(\rho, n, j)\right) \\
& \quad \times M_{n}(j, q(w, p))\left(\frac{s_{n+k+1}}{s_{n}}\right)^{(p)}\left(\tilde{\alpha}_{w}\right) .
\end{aligned}
$$


Let us transform the triple sum on the right side of the last expression

$$
\begin{aligned}
& \frac{1}{\Lambda_{n}} \sum_{w=1}^{\gamma} \sum_{p=0}^{k_{w}-1} \sum_{j=1}^{k}(-1)^{j+q(w, p)}\left(\left[F Q_{m}\right]_{n+j}-\eta(\rho, n, j)\right) M_{n}(j, q(w, p)) \\
& \quad \times\left(\frac{s_{n+k+1}}{s_{n}}\right)^{(p)}\left(\tilde{\alpha}_{w}\right) \\
& =\frac{1}{\Lambda_{n}} \sum_{j=1}^{k}\left(\left[F Q_{m}\right]_{n+j}-\eta(\rho, n, j)\right) \sum_{w=1}^{\gamma} \sum_{p=0}^{k_{w}-1}(-1)^{j+q(w, p)} M_{n}(j, q(w, p)) \\
& \quad \times\left(\frac{s_{n+k+1}}{s_{n}}\right)^{(p)}\left(\tilde{\alpha}_{w}\right) \\
& =\frac{1}{\Lambda_{n}} \sum_{j=1}^{k}\left(\left[F Q_{m}\right]_{n+j}-\eta(\rho, n, j)\right) \tilde{\Lambda}_{n}(j, k+1) \\
& =\frac{1}{\Lambda_{n}} \sum_{j=1}^{k}\left[F Q_{m}\right]_{n+j} \tilde{\Lambda}_{n}(j, k+1)-\frac{1}{\Lambda_{n}} \sum_{j=1}^{k} \eta(\rho, n, j) \tilde{\Lambda}_{n}(j, k+1),
\end{aligned}
$$

where we denote by $\tilde{\Lambda}_{n}(j, k+1)$ the determinant obtained from $\Lambda_{n}$ replacing the $j$ th row by the row

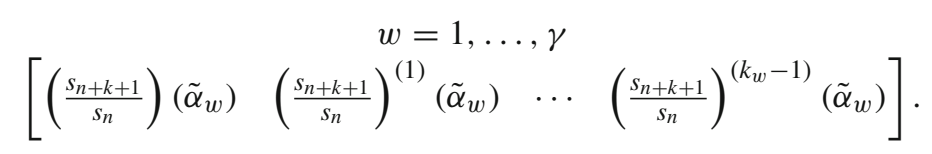

Define

$$
a_{j, n}:=-\frac{\tilde{\Lambda}_{n}(j, k+1)}{\Lambda_{n}}, \quad \text { for } \quad j=1, \ldots, k, \quad \text { and } \quad a_{k+1, n}:=1
$$

Therefore, we have

$$
\sum_{j=1}^{k+1} a_{j, n}\left[F Q_{m}\right]_{n+j}=\sum_{j=1}^{k+1} a_{j, n} \eta(\rho, n, j) .
$$

Let us obtain some lower and upper bounds for $\left|a_{j, n}\right|$, for $j=1, \ldots, k$. Since 


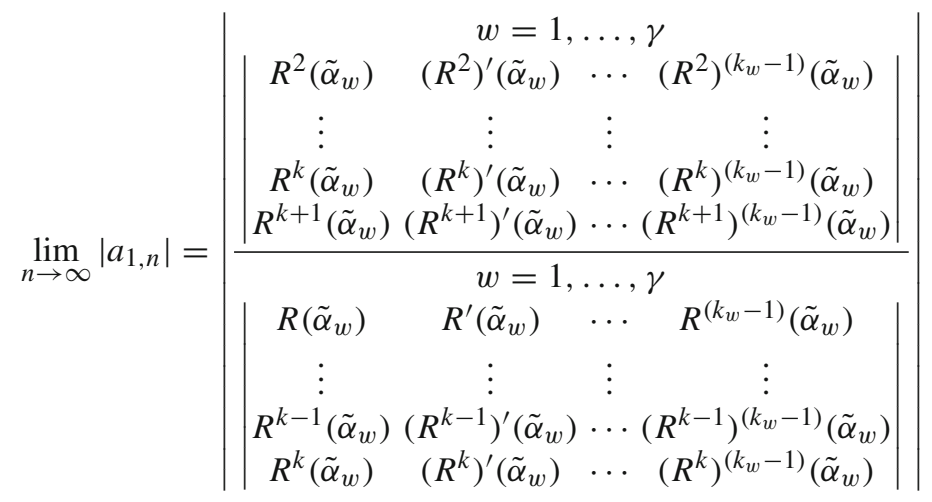

$$
\begin{aligned}
& =\frac{\left|\prod_{w=1}^{\gamma}\left(\left(k_{w}-1\right) ! !\left(\Phi^{\prime}\left(\tilde{\alpha}_{w}\right)\right)^{k_{w}\left(k_{w}-1\right) / 2} \Phi\left(\tilde{\alpha}_{w}\right)^{-k_{w}\left(k_{w}+1\right)}\right) \prod_{1 \leq i<j \leq \gamma}\left(1 / \Phi\left(\tilde{\alpha}_{j}\right)-1 / \Phi\left(\tilde{\alpha}_{i}\right)\right)^{k_{i} k_{j}}\right|}{\left|\prod_{w=1}^{\gamma}\left(\left(k_{w}-1\right) ! !\left(\Phi^{\prime}\left(\tilde{\alpha}_{w}\right)\right)^{k_{w}\left(k_{w}-1\right) / 2} \Phi\left(\tilde{\alpha}_{w}\right)^{-k_{w}^{2}}\right) \prod_{1 \leq i<j \leq \gamma}\left(1 / \Phi\left(\tilde{\alpha}_{j}\right)-1 / \Phi\left(\tilde{\alpha}_{i}\right)\right)^{k_{i} k_{j}}\right|} \\
& =\prod_{w=1}^{\gamma}\left|\left(\Phi\left(\tilde{\alpha}_{w}\right)\right)^{-k_{w}}\right| \neq 0,
\end{aligned}
$$

it follows that for $n$ sufficiently large,

$$
0<c_{2} \leq\left|a_{1, n}\right| \leq c_{3}
$$

Analogously, one obtains

$$
\left|a_{j, n}\right| \leq c_{4}, \quad j=2,3, \ldots, k .
$$

The inequalities (28) and (29) will be used later.

In (27), we replace the index $n$ by the indices $n+v, v=0,1, \ldots, v_{0}-1$, where $v_{0}$ is an arbitrary natural number greater than $3 k+1$. Then, we have

$$
\sum_{j=1}^{k+1} a_{j, n+\nu}\left[F Q_{m}\right]_{n+\nu+j}=\sum_{j=1}^{k+1} a_{j, n+\nu} \eta(\rho, n+\nu, j), \quad v=0,1, \ldots, \nu_{0}-1 .
$$

We rewrite the system of Eq. (30) in the following form

$$
\sum_{j=1}^{\min \left(v_{0}-v, k+1\right)} a_{j, n+v}\left[F Q_{m}\right]_{n+v+j}=B_{n+v}(\rho), \quad v=0,1, \ldots, v_{0}-1
$$


where

$$
\begin{aligned}
B_{n+v}(\rho):= & \sum_{j=1}^{k+1} a_{j, n+v} \eta(\rho, n+v, j), \quad v=0,1, \ldots, v_{0}-k-1, \\
B_{n+v}(\rho):= & \sum_{j=1}^{k+1} a_{j, n+v} \eta(\rho, n+v, j)-\sum_{j=v_{0}-v+1}^{k+1} a_{j, n+v}\left[F Q_{m}\right]_{n+v+j}, \\
& v=v_{0}-k, \ldots, v_{0}-1 .
\end{aligned}
$$

We view this as a system of $v_{0}$ equations on the $v_{0}$ unknowns $\left[F Q_{m}\right]_{n+1}, \ldots,[F$ $\left.Q_{m}\right]_{n+v_{0}}$. Notice that the matrix corresponding to the system is upper triangular and its determinant equals

$$
\Lambda_{n}^{*}\left(v_{0}\right)=\prod_{\nu=0}^{\nu_{0}-1} a_{1, n+\nu} \neq 0
$$

for all $n$ sufficiently large (see (28)). Therefore, $\left[F Q_{m}\right]_{n+1}=\Lambda_{n}^{*}\left(v_{0}, 1\right) / \Lambda_{n}^{*}\left(v_{0}\right)$, where $\Lambda_{n}^{*}\left(\nu_{0}, 1\right)$ is the determinant obtained replacing the first column of the determinant of the system by the column $\left[B_{n}(\rho) \cdots B_{n+v_{0}-1}(\rho)\right]^{T}$. Expanding $\Lambda_{n}^{*}\left(\nu_{0}, 1\right)$ by the first column, we get

$$
\Lambda_{n}^{*}\left(\nu_{0}, 1\right)=\sum_{\nu=0}^{\nu_{0}-1}(-1)^{v} B_{n+v}(\rho) D\left(n, v_{0}, v\right)
$$

where $D\left(n, v_{0}, v\right)$ is the $(v+1,1)$ th minor of $\Lambda_{n}^{*}\left(v_{0}, 1\right)$. Moreover, it is easy to check that

$$
D\left(n, v_{0}, v\right)=D(n, v+1, v) \prod_{j=v+1}^{\nu_{0}-1} a_{1, n+j}
$$

and we denote $D(n, v):=D(n, v+1, v)$. Therefore, by (31), we have

$$
\left[Q_{m} F\right]_{n+1}=\sum_{\nu=0}^{\nu_{0}-1}(-1)^{\nu} B_{n+v}(\rho) \frac{D\left(n, v_{0}, v\right)}{\prod_{j=0}^{\nu_{0}-1} a_{1, n+j}}=\sum_{\nu=0}^{\nu_{0}-1}(-1)^{v} B_{n+v}(\rho) \frac{D(n, v)}{\prod_{j=0}^{v} a_{1, n+j}}
$$

From (28) and (29), we get

$$
\left|\frac{D(n, v)}{\prod_{j=0}^{v} a_{1, n+j}}\right| \leq c_{5}, \quad v=0,1, \ldots, 3 k .
$$


Our next goal is to estimate

$$
\left|\frac{D(n, v)}{\prod_{j=0}^{v} a_{1, n+j}}\right|, \quad v=3 k+1,3 k+2, \ldots, v_{0}-1 .
$$

For this purpose, we expand the determinant $D(n, v)$ along the first row. We have

$$
D(n, v)=\sum_{p=1}^{k}(-1)^{p+1} a_{p+1, n} M_{n}(v, 1, p),
$$

where $M_{n}(v, 1, p)$ is the $(1, p)$ th minor of $D(n, v)$. It is easy to check that for $v \geq k+1$,

$$
M(v, 1, p)=\frac{1}{a_{1, n}} \prod_{j=0}^{p-1} a_{1, n+j} D(n+p, v-p) .
$$

Hence,

$$
D(n, v)=\frac{1}{a_{1, n}} \sum_{p=1}^{k}(-1)^{p+1} a_{p+1, n} \prod_{j=0}^{p-1} a_{1, n+j} D(n+p, v-p) .
$$

Replacing $n$ by $n+r$ and $v$ by $v-r$, where $r=0,1,2, \ldots, v-2 k$, we obtain the following relations

$$
a_{1, n+r} D(n+r, v-r)=\sum_{p=1}^{k}(-1)^{p+1} a_{p+1, n+r} \prod_{j=r}^{r+p-1} a_{1, n+j} D(n+r+p, v-r-p) .
$$

Dividing both sides by $\prod_{j=r}^{v-1} a_{1, n+j}$, we get

$$
a_{1, n+r} \frac{D(n+r, v-r)}{\prod_{j=r}^{v-1} a_{1, n+j}}=\sum_{p=1}^{k}(-1)^{p+1} a_{p+1, n+r} \frac{D(n+r+p, v-r-p)}{\prod_{j=r+p}^{v-1} a_{1, n+j}} .
$$

For fixed $n$ and $v$, the quantity

$$
V_{\nu-(r+p)}:=(-1)^{r+p} \frac{D(n+p+r, v-r-p)}{\prod_{j=r+p}^{v-1} a_{1, n+j}}
$$

depends only on the sum $r+p$. With this notation, (33) can be rewritten as

$$
\sum_{p=0}^{k} a_{p+1, n+r} V_{v-(r+p)}=0, \quad r=0,1, \ldots, v-2 k
$$

Setting $\tilde{\Lambda}_{n+r}(k+1, k+1)=-\Lambda_{n+r}$, we bring these relations to the form 


$$
\sum_{p=0}^{k}\left(-\frac{\tilde{\Lambda}_{n+r}(p+1, k+1)}{\Lambda_{n+r}}\right) V_{\nu-(r+p)}=0
$$

or, what is the same,

$$
\sum_{p=0}^{k} \tilde{\Lambda}_{n+r}(p+1, k+1) V_{\nu-(r+p)}=0, \quad r=0,1, \ldots, v-2 k .
$$

Let us show that the Eq. (34) are equivalent to

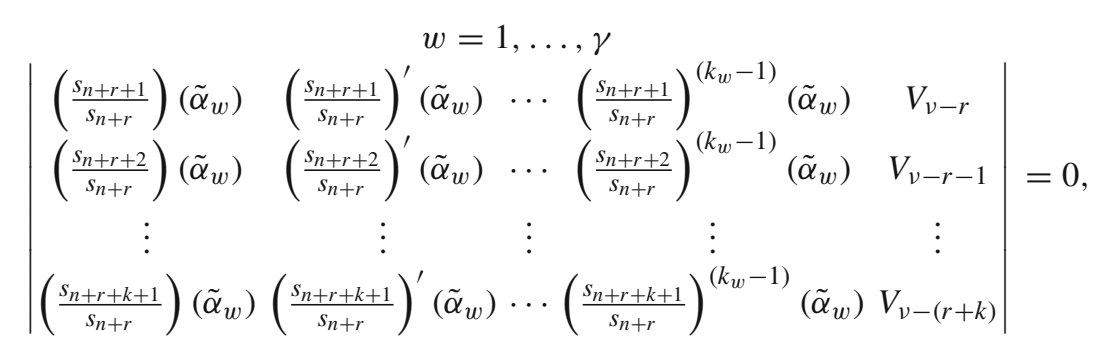

for $r=0,1, \ldots, v-2 k$ (this expression represents the determinant of order $k+1$ in which the indicated group of columns, evaluated at $\tilde{\alpha}_{w}$, are successively written out for $w=1, \ldots, \gamma$ and the last column is $\left.\left[\begin{array}{llll}V_{\nu-r} & V_{\nu-r-1} & \cdots & V_{\nu-(r+k)}\end{array}\right]^{T}\right)$. In fact, expanding (35) along the last column, we have

$$
\sum_{q=1}^{k+1}(-1)^{k+q+1} \tilde{M}_{n}^{r}(q, k+1) V_{v-(r+q-1)}=0,
$$

where $\tilde{M}_{n}^{r}(q, k+1)$ is the $(q, k+1)$ th minor of the determinant in (35). Moreover, it is easy to check that

$$
\tilde{M}_{n}^{r}(q, k+1)=(-1)^{k-q} \tilde{\Lambda}_{n+r}(q, k+1) .
$$

Therefore, setting $q=p+1$ in (34), we obtain

$$
\begin{aligned}
0 & =-\sum_{q=1}^{k+1} \tilde{\Lambda}_{n+r}(q, k+1) V_{\nu-(r+q-1)} \\
& =\sum_{q=1}^{k+1}(-1)^{k+q+1}(-1)^{k-q} \tilde{\Lambda}_{n+r}(q, k+1) V_{\nu-(r+q-1)} \\
& =\sum_{q=1}^{k+1}(-1)^{k+q+1} \tilde{M}_{n}^{r}(q, k+1) V_{\nu-(r+q-1)}
\end{aligned}
$$

as we needed to show. 
Let us transform (35) further. By the Leibniz rule, we have for all $j \geq 1$ and $p \geq 0$,

$$
\left(\frac{s_{n+r+j}}{s_{n+v+1}}\right)^{(p)}=\sum_{i=0}^{p}\left(\begin{array}{l}
p \\
i
\end{array}\right)\left(\frac{s_{n+r}}{s_{n+v+1}}\right)^{(p-i)}\left(\frac{s_{n+r+j}}{s_{n+r}}\right)^{(i)}
$$

Notice that the factors of $\left(\frac{s_{n+r+j}}{s_{n+r}}\right)^{(i)}$ do not depend on $j$. Consequently, taking column operations on the determinant in (35) and having in mind that the determinant equals zero, we obtain the system (35) is equivalent to

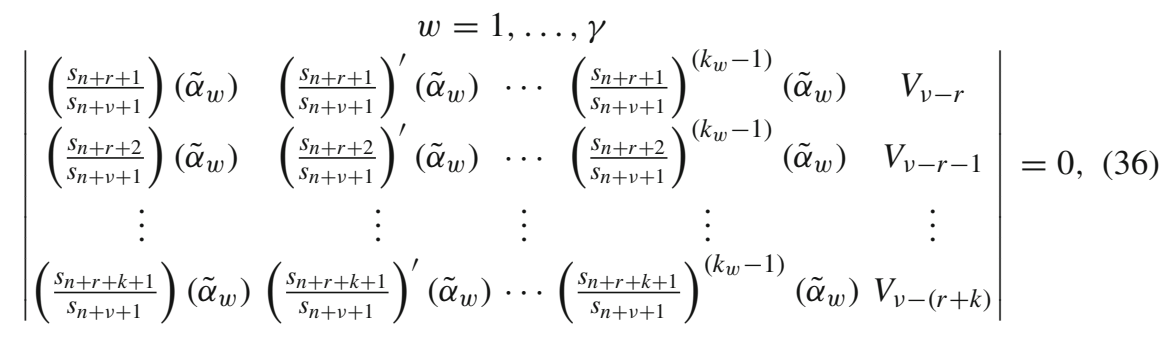

for $r=0, \ldots, v-2 k$.

We consider (36) as a linear system of $v-2 k+1$ equations with $v-k+1$ unknowns $V_{k}, \ldots, V_{v}$. The rank of this system is $v-2 k+1$ for $n$ sufficiently large. Thus, the null space has dimension $k$. Therefore, every solution of (36) can be written as a unique linear combination of $k$ linearly independent solutions $W_{1}(n), \ldots, W_{k}(n)$. The structure of (36) easily reveals that for each $w=1, \ldots, \gamma$ and $p=0, \ldots, k_{w}-1$

$W_{j(w, p)}(n)=\left[\left(\frac{s_{n+h+1}}{s_{n+v+1}}\left(\tilde{\alpha}_{w}\right)\right)^{(p)}\right]_{h=0, \ldots, v-k}^{T}, \quad j(w, p)=\sum_{l=0}^{w-1} k_{l}+p+1, \quad k_{0}=0$,

is a solution of the homogeneous linear system of Eq. (36). Moreover, they are linearly independent (for all sufficiently large $n$ ) because using (12)

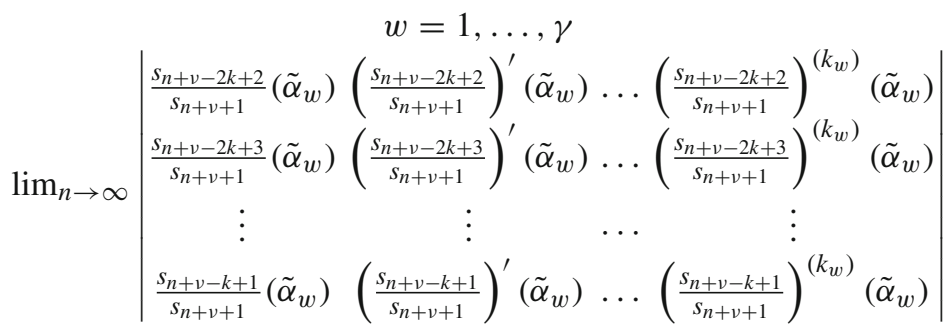

$$
\begin{aligned}
& =(-1)^{(k-1) k / 2} \prod_{w=1}^{\gamma}\left(k_{w}-1\right) ! !\left(\Phi^{k_{w} k}\right)\left(\tilde{\alpha_{w}}\right)\left(\Phi^{\prime}\left(\tilde{\alpha_{w}}\right)\right)^{\frac{\left(k_{w}-1\right) k_{w}}{2}} \\
& \times \prod_{1 \leq i<j \leq \gamma}\left(\Phi\left(\tilde{\alpha}_{j}\right)-\Phi\left(\tilde{\alpha}_{i}\right)\right)^{k_{i} k_{j}} \neq 0
\end{aligned}
$$


Since

$$
V_{\nu-(r+p)}:=(-1)^{r+p} \frac{D(n+p+r, v-r-p)}{\prod_{j=r+p}^{v-1} a_{1, n+j}},
$$

there exists a unique set of coefficients $C_{1}(n), \ldots, C_{k}(n)$ such that

$$
\left[(-1)^{h} \frac{D(n+h, v-h)}{\prod_{j=h}^{v-1} a_{1, n+j}}\right]_{h=0, \ldots, v-k}=\sum_{j=1}^{k} C_{j}(n) W_{j}(n)
$$

Thus,

$$
\begin{gathered}
\sum_{w=1}^{\gamma} \sum_{p=0}^{k_{w}-1} c_{n, v}(w, p)\left(\frac{s_{n+h+1}}{s_{n+v+1}}\right)^{(p)}\left(\tilde{\alpha}_{w}\right)=(-1)^{h} \frac{D(n+h, v-h)}{\prod_{\tau=h}^{v-1} a_{1, n+\tau}} \\
h=0, \ldots, v-k
\end{gathered}
$$

where the constants $c_{n, v}(w, p)$ are uniquely determined.

To estimate the $c_{n, v}(w, p), w=1, \ldots, \gamma, p=0, \ldots, k_{w}-1$, we use the linear system of equations

$$
\sum_{w=1}^{\gamma} \sum_{p=0}^{k_{w}-1} c_{n, v}(w, p)\left(\frac{s_{n+h+1}}{s_{n+v+1}}\right)^{(p)}\left(\tilde{\alpha}_{w}\right)=(-1)^{h} \frac{D(n+h, v-h)}{\prod_{\tau=h}^{v-1} a_{1, n+\tau}}
$$

corresponding to $h=v-2 k+1, \ldots, v-k$. From (37), it follows that the determinant of this system is different from zero for all sufficiently large $n$. From (28) and (29), it is not difficult to verify that

$$
\left|\frac{D(n+h, v-h)}{\prod_{\tau=h}^{v-1} a_{1, n+\tau}}\right| \leq c_{6}, \quad h=v-2 k+1, \ldots, v-k,
$$

From (12) and the Weierstrass theorem, we have

$$
\lim _{n \rightarrow \infty}\left(\frac{s_{n+h+1}(z)}{s_{n+v+1}(z)}\right)^{(p)}=\left(\Phi^{\nu-h}(z)\right)^{(p)}
$$

uniformly inside $\overline{\mathbb{C}} \backslash E$. Therefore, the coefficients of system (39) remain uniformly bounded with respect to $n$ or $v$ since in those equations $k \leq v-h \leq 2 k-1$. Applying Cramer's rule and (40), it follows that

$$
\left|c_{n, v}(w, p)\right| \leq c_{7}, \quad w=1, \ldots, \gamma, \quad p=0, \ldots, k_{w}-1
$$


where $c_{7}$ does not depend on $n$ or $v$. Taking $h=0$ in (38), we have

$$
\frac{D(n, v)}{\prod_{\tau=0}^{v-1} a_{1, n+\tau}}=\sum_{w=1}^{\gamma} \sum_{p=0}^{k_{w}-1} c_{n, v}(w, p)\left(\frac{s_{n+1}}{s_{n+v+1}}\right)^{(p)}\left(\tilde{\alpha}_{w}\right) .
$$

From (42), (43), and (41) with $h=0$, it follows that for any $\varepsilon>0$ there exists $n_{0}$ such that for $n \geq n_{0}$

$$
\left|\frac{D(n, v)}{\prod_{\tau=0}^{v-1} a_{1, n+\tau}}\right| \leq c_{8}\left(\sigma_{\gamma}+\varepsilon\right)^{v}, \quad v=0, \ldots, v_{0}-1 .
$$

(Notice that using Cauchy's integral formula it is easy to prove that $\left|\left(\Phi^{v}\left(\widetilde{\alpha}_{w}\right)\right)^{(p)}\right| \leq$ $\left.c_{9}\left|\Phi^{\nu}\left(\sigma_{\gamma}+\varepsilon\right)\right|\right)$. Now, (28), (32), and (44) give

$$
\begin{aligned}
\left|\left[Q_{m} F\right]_{n+1}\right| & \leq \sum_{\nu=0}^{\nu_{0}-1}\left|B_{n+v}(\rho)\right|\left|\frac{D(n, \nu)}{\prod_{\tau=0}^{v-1} a_{1, n+\tau}}\right| \frac{1}{\left|a_{1, n+\nu}\right|} \\
& \leq c_{10} \sum_{\nu=0}^{\nu_{0}-1}\left|B_{n+v}(\rho)\right|\left(\sigma_{\gamma}+\varepsilon\right)^{\nu} .
\end{aligned}
$$

Next, let us bound $\left|B_{n+v}(\rho)\right|$. Take $\varepsilon>0$ such that $\sigma_{\gamma}+\varepsilon<\rho-\varepsilon$ and $\delta<\delta^{\prime}<1$. From (9), (14), and (19), we have for all sufficiently large $n$

$$
|\eta(\rho, n, j)| \leq c_{11}\left(\delta^{\prime}\right)^{n} \frac{1}{(\rho-\varepsilon)^{n+j}},
$$

and

$$
\left|\left[Q_{m} F\right]_{n+v+j}\right| \leq \frac{c_{12}}{(\rho-\varepsilon)^{n+v+j}} .
$$

Thus, from (28), (29) and the definition of $B_{n+v}(\rho)$, we obtain

$$
\begin{aligned}
& \left|B_{n+v}(\rho)\right| \leq c_{13}\left(\frac{\delta^{\prime}}{\rho-\varepsilon}\right)^{n+v} \sum_{j=1}^{k+1} \frac{1}{(\rho-\varepsilon)^{j}} \\
& \quad=c_{14}\left(\frac{\delta^{\prime}}{\rho-\varepsilon}\right)^{n}\left(\frac{1}{\rho-\varepsilon}\right)^{v}, \quad v=0,1, \ldots, v_{0}-k-1,
\end{aligned}
$$

and

$$
\left|B_{n+v}(\rho)\right| \leq c_{14}\left(\frac{\delta^{\prime}}{\rho-\varepsilon}\right)^{n}\left(\frac{1}{\rho-\varepsilon}\right)^{v}+\sum_{j=v_{0}-v+1}^{k+1} \frac{c_{15}}{(\rho-\varepsilon)^{n+v+j}}
$$




$$
\begin{aligned}
& \leq c_{14}\left(\frac{\delta^{\prime}}{\rho-\varepsilon}\right)^{n}\left(\frac{1}{\rho-\varepsilon}\right)^{v}+c_{16} \frac{1}{(\rho-\varepsilon)^{n+v}} \\
& \leq \frac{c_{17}}{(\rho-\varepsilon)^{n+v}}, \quad v=v_{0}-k, \ldots, v_{0}-1 .
\end{aligned}
$$

Applying (46) and (47) to (45), we have

$\left|\left[Q_{m} F\right]_{n+1}\right| \leq c_{18}\left(\left(\frac{\delta^{\prime}}{\rho-\varepsilon}\right)^{n} \sum_{\nu=0}^{\nu_{0}-k-1}\left(\frac{\sigma_{\gamma}+\varepsilon}{\rho-\varepsilon}\right)^{\nu}+\frac{1}{(\rho-\varepsilon)^{n}} \sum_{\nu=\nu_{0}-k}^{\nu_{0}-1}\left(\frac{\sigma_{\gamma}+\varepsilon}{\rho-\varepsilon}\right)^{\nu}\right)$

Setting $\theta=\left(\sigma_{\gamma}+\varepsilon\right) /(\rho-\varepsilon)<1$, we find that

$$
\left|\left[Q_{m} F\right]_{n+1}\right| \leq c_{19}\left(\left(\frac{\delta^{\prime}}{\rho-\varepsilon}\right)^{n+1} \sum_{\nu=0}^{\infty} \theta^{\nu}+\frac{1}{(\rho-\varepsilon)^{n}} \sum_{\nu=\nu_{0}-k}^{\infty} \theta^{\nu}\right)
$$

Letting $v_{0} \rightarrow \infty$, we obtain

$$
\left|\left[Q_{m} F\right]_{n+1}\right| \leq c_{20}\left(\frac{\delta^{\prime}}{\rho-\varepsilon}\right)^{n+1},
$$

and

$$
\varlimsup_{n \rightarrow \infty}\left|\left[Q_{m} F\right]_{n+1}\right|^{1 /(n+1)} \leq \frac{\delta^{\prime}}{\rho-\varepsilon} .
$$

Making $\varepsilon \rightarrow 0, \delta^{\prime} \rightarrow \delta$, and $\rho \rightarrow \rho_{m}(F)$, we obtain the claim that

$$
\varlimsup_{n \rightarrow \infty}\left|\left[Q_{m} F\right]_{n+1}\right|^{1 /(n+1)} \leq \frac{\delta}{\rho_{m}(F)}
$$

From this and (13), if follows that the function $Q_{m} F$ is holomorphic in $D_{\rho_{m}(F) / \delta}$. Thus, $F$ is meromorphic with at most $m$ poles on $D_{\rho_{m}(F) / \delta}$ which contradicts the definition of $\rho_{m}(F)$ unless $\rho_{m}(F)=\infty$.

In the final step, we show that if $F$ is meromorphic in $\mathbb{C}$ and has $k<m$ poles, then $F$ is a rational function. In fact, in that case

$$
F:=F^{*}+R_{k}
$$

where $F^{*}$ is an entire function and $R_{k}$ is a rational function with $k$ poles at $\lambda_{1}, \ldots, \lambda_{k}$. Applying the residue theorem and arguing as in (18), we obtain 


$$
\begin{aligned}
{\left[R_{k} Q_{n, m}^{\mu}\right]_{n+b}=} & \frac{1}{2 \pi i} \int_{\Gamma_{\rho}} R_{k}(t) Q_{n, m}^{\mu}(t) s_{n+b}(t) \mathrm{d} t-\sum_{w=1}^{\gamma} \operatorname{res}\left(R_{k} Q_{n, m}^{\mu} s_{n+b}, \tilde{\alpha}_{w}\right) \\
= & \frac{1}{2 \pi i} \int_{\Gamma_{\rho}} R_{k}(t) Q_{n, m}^{\mu}(t) s_{n+b}(t) \mathrm{d} t \\
& -\sum_{w=1}^{\gamma} \sum_{p=0}^{k_{w}-1} \xi_{n}(w, p)\left(\frac{s_{n+b}}{s_{n+1}}\right)^{(p)}\left(\tilde{\alpha_{w}}\right),
\end{aligned}
$$

where $\rho>\sigma_{\gamma}$ and

$\xi_{n}(w, p)=\frac{1}{\left(k_{w}-1\right) !}\left(\begin{array}{c}k_{w}-1 \\ p\end{array}\right) \lim _{z \rightarrow \tilde{\alpha}_{w}}\left(\left(z-\tilde{\alpha}_{w}\right)^{k_{w}} R_{k}(z) Q_{n, m}^{\mu}(z) s_{n+1}(z)\right)^{\left(k_{w}-1-p\right)}$.

Since $s_{n+b}$ has a zero of order $n+b+1$ at infinity and $\operatorname{deg}\left(Q_{n, m}^{\mu}\right) \leq m$, for $n$ sufficiently large, we have

$$
\frac{1}{2 \pi i} \int_{\Gamma_{\rho}} R_{k}(t) Q_{n, m}^{\mu}(t) s_{n+b}(t) \mathrm{d} t=0 .
$$

By the definition of Padé-orthogonal approximants,

$$
0=\left[F Q_{n, m}^{\mu}\right]_{n+b}=\left[F^{*} Q_{n, m}^{\mu}\right]_{n+b}+\left[R_{k} Q_{n, m}^{\mu}\right]_{n+b}, \quad b=1, \ldots, m .
$$

Since $k+1 \leq m$, using (48) and (49), we have

$$
\left[F^{*} Q_{n, m}^{\mu}\right]_{n+b}=\sum_{w=1}^{\gamma} \sum_{p=0}^{k_{w}-1} \xi_{n}(w, p)\left(\frac{s_{n+b}}{s_{n+1}}\right)^{(p)}\left(\tilde{\alpha_{w}}\right), \quad b=1, \ldots, k+1 .
$$

Arguing as above in the deduction of (21)-(26), we obtain

$$
\left[F^{*} Q_{n, m}^{\mu}\right]_{n+1}=\sum_{j=2}^{k+1} a_{j, n}\left[F^{*} Q_{n, m}^{\mu}\right]_{n+j},
$$

where $a_{j, n}:=\Lambda_{n+1}(j-1,1) / \Lambda_{n+1}, \Lambda_{n+1}$ is matrix (22) with $n$ replaced by $n+1$, and $\Lambda_{n+1}(j-1,1)$ is the determinant obtained from $\Lambda_{n+1}$ replacing row $j-1$ by the row

$$
\left.\begin{array}{llllll}
\multicolumn{5}{c}{w=1, \ldots, \gamma} & \\
{[1} & 0 & 0 & 0 & \ldots & 0
\end{array}\right] .
$$

It is easy to verify that $\left|a_{j, n}\right| \leq c_{21}$, for all $j=2, \ldots, k+1$. Therefore,

$$
\left|\left[F^{*} Q_{n, m}^{\mu}\right]_{n+1}\right| \leq c_{21} \sum_{j=2}^{k+1}\left|\left[F^{*} Q_{n, m}^{\mu}\right]_{n+j}\right| .
$$


Let

$$
Q_{n, m}^{\mu}(z):=z^{m}+\sum_{j=0}^{m-1} q_{n, j} z^{j}
$$

and

$$
F^{*}(z):=\sum_{\nu=0}^{\infty} F_{v}^{*} p_{v}(z),
$$

where $F_{v}^{*}:=\left\langle F^{*}, p_{v}\right\rangle_{\mu}$. Note that the series $\sum_{\nu=0}^{\infty} F_{v}^{*} p_{\nu}$ converges to $F^{*}$ uniformly inside $\mathbb{C}$ and $\lim _{v \rightarrow \infty}\left|F_{v}^{*}\right|^{1 / v}=0$ because $F^{*}$ is an entire function. Therefore, for all $b=1, \ldots, k+1$,

$$
\begin{aligned}
{\left[Q_{n, m}^{\mu} F^{*}\right]_{n+b} } & =\left\langle Q_{n, m}^{\mu} F^{*}, p_{n+b}\right\rangle_{\mu}=\left\langle z^{m} F^{*}, p_{n+b}\right\rangle_{\mu}+\sum_{j=0}^{m-1} q_{n, j}\left\langle z^{j} F^{*}, p_{n+b}\right\rangle_{\mu} \\
& =\sum_{\nu=0}^{\infty} F_{\nu}^{*}\left\langle z^{m} p_{\nu}, p_{n+b}\right\rangle_{\mu}+\sum_{j=0}^{m-1} q_{n, j} \sum_{\nu=0}^{\infty} F_{\nu}^{*}\left\langle z^{j} p_{\nu}, p_{n+b}\right\rangle_{\mu} \\
& =\sum_{\nu=n+b-m}^{\infty} F_{\nu}^{*}\left\langle z^{m} p_{\nu}, p_{n+b}\right\rangle_{\mu}+\sum_{j=0}^{m-1} q_{n, j} \sum_{\nu=n+b-j}^{\infty} F_{\nu}^{*}\left\langle z^{j} p_{\nu}, p_{n+b}\right\rangle_{\mu} .
\end{aligned}
$$

By the Cauchy-Schwarz inequality and the orthonormality of $p_{v}$, for all $n, v, b \in \mathbb{N}$ and $j=1, \ldots, m$,

$$
\left|\left\langle z^{j} p_{\nu}, p_{n+b}\right\rangle_{\mu}\right| \leq c_{22} .
$$

Using (9), it follows that $\left|q_{n, j}\right| \leq c_{23}$ and therefore

$$
\left|\left[Q_{n, m}^{\mu} F^{*}\right]_{n+b}\right| \leq c_{24} \sum_{\nu=n+b-m}^{\infty}\left|F_{v}^{*}\right| \leq c_{24} \sum_{\nu=n+2-m}^{\infty}\left|F_{\nu}^{*}\right|, \quad b=2, \ldots k+1(51)
$$

Moreover,

$$
\begin{aligned}
{\left[Q_{n, m}^{\mu} F^{*}\right]_{n+1}=} & \sum_{\nu=n+1-m}^{\infty} F_{\nu}^{*}\left\langle z^{m} p_{v}, p_{n+1}\right\rangle_{\mu}+\sum_{j=0}^{m-1} q_{n, j} \sum_{\nu=n+1-j}^{\infty} F_{v}^{*}\left\langle z^{j} p_{\nu}, p_{n+1}\right\rangle_{\mu} \\
= & \frac{\kappa_{n+1-m}}{\kappa_{n+1}} F_{n+1-m}^{*}+\sum_{\nu=n+2-m}^{\infty} F_{v}^{*}\left\langle z^{m} p_{v}, p_{n+1}\right\rangle_{\mu} \\
& +\sum_{j=0}^{m-1} q_{n, j} \sum_{\nu=n+1-j}^{\infty} F_{v}^{*}\left\langle z^{j} p_{v}, p_{n+1}\right\rangle_{\mu}
\end{aligned}
$$


Combining (50), (51), and (52), we have

$$
\frac{\kappa_{n+1-m}}{\kappa_{n+1}}\left|F_{n+1-m}^{*}\right| \leq c_{25} \sum_{\nu=n+2-m}^{\infty}\left|F_{\nu}^{*}\right| .
$$

By Lemma 2,

$$
\lim _{n \rightarrow \infty} \frac{\kappa_{n+1-m}}{\kappa_{n+1}}=\operatorname{cap}(E)^{m}>0
$$

therefore, there exists $n_{1} \geq 1$ such that for all $n \geq n_{1}$,

$$
\frac{\kappa_{n+1-m}}{\kappa_{n+1}} \geq c_{26}>0
$$

Setting $N=n+1-m$, we obtain

$$
\left|F_{N}^{*}\right| \leq c_{27} \sum_{\nu=N+1}^{\infty}\left|F_{N}^{*}\right|, \quad N \geq N_{0}
$$

By Lemma 3, there exist $N_{1} \in \mathbb{N}$ such that $F_{N}^{*}=0$ for all $N \geq N_{1}$. Therefore, $F^{*}$ is a polynomial and $F$ is a rational function with at most $k$ poles. However, in this case, it is easy to see from (4) that under appropriate column operations $\Delta_{n, m}(F, \mu)=0$ for all $n$ sufficiently large. This contradicts the assumption that for all $n$ sufficiently large, $\operatorname{deg}\left(Q_{n, m}^{\mu}\right)=m$. Consequently, $F$ has $m$ poles in $D_{\rho_{m}(F)}$.

By Theorem A, we conclude that $\lambda_{1}, \ldots, \lambda_{m}$ are poles of $F$ in $D_{\rho_{m}(F)}$. To prove (10), let us consider the region $D_{\rho_{m-1}(F)}$. Notice that $\rho_{m-1}(F):=$ $\max _{j=1, \ldots, m}\left|\Phi\left(\lambda_{j}\right)\right|$. Clearly, $F$ has less than $m$ poles in $D_{\rho_{m-1}(F)}$. Repeating the proof above we obtain $\varlimsup_{n \rightarrow \infty}\left[Q_{m} F\right]_{n}^{1 / n} \leq \delta / \rho_{m-1}(F)$. This implies that $F$ is meromorphic with at most $m$ poles in $D_{\rho_{m-1}(F) / \delta}$. From the definition of $\rho_{m}(F)$ this implies that

$$
\rho_{m}(F) \geq \frac{1}{\delta} \max _{j=1 \ldots, m}\left|\Phi\left(\lambda_{j}\right)\right|
$$

This completes the proof.

\section{References}

1. Baker, G.A., Graves-Morris, P.: Padé approximants, 2nd edn. In: Encyclopedia of Mathematics and its Applications, vol. 59. Cambridge University, Cambridge (1996)

2. Rolanía, D.B., López Lagomasino, G.: Ratio asymptotics for polynomials orthogonal on arcs of the unit circle. Constr. Approx. 15, 1-31 (1999)

3. Rolanía, D.B., López Lagomasino, G., Saff, E.B.: Asymptotics of orthogonal polynomials inside the unit circle and Szegő-Padé approximants. J. Comput. Appl. Math. 133, 171-181 (2001)

4. Rolanía, D.B., López Lagomasino, G., Saff, E.B.: Determining radii of meromorphy via orthogonal polynomials on the unit circle. J. Approx. Theory 124, 263-281 (2003) 
5. Hernández, M.B., López Lagomasino, G.: Ratio and relative asymptotics of polynomials orthogonal on an arc of the unit circle. J. Approx. Theory 92, 216-244 (1998)

6. Bosuwan, N., López Lagomasino, G., Saff, E.B.: Determining singularities using row sequences of Padé-orthogonal approximants. Jaen J. Approx. 5, 179-208 (2013)

7. Buslaev, V.I.: On the Fabry ratio theorem for orthogonal series. Complex analysis and applications. Proc. Steklov Inst. Math. 253, 8-21 (2006)

8. Buslaev, V.I.: An analogue of Fabry's theorem for generalized Padé approximants. Math. Sb. 200, 39-106 (2009)

9. Cacoq, J., López Lagomasino., G.: Convergence of row sequences of simultaneous Fourier-Padé approximation. Jaen J. Approx. 4, 101-120 (2012)

10. Cacoq, J., de la Calle Ysern, B., López Lagomasino, G.: Direct and inverse results on row sequences of Hermite-Padé approximants. Constr. Approx. 38, 133-160 (2013)

11. Carleman, T.: Über die Approximation analytischer Funktionen durch lineare Aggregate von vorgegebenen Potenzen. Ark. Mat. Astron. Fys. 17, 215-244 (1923)

12. Cheney, E.W.: Introduction to Approximation Theory. McGraw-Hill, New York (1966)

13. Fleischer, J.: Generalizations of Padé Approximants. Los Alamos Scientific Laboratory, New Mexico (1972)

14. Goluzin, G.M.: Geometric Theory of Functions of a Complex Variable. Transl. of Math. Monographs, vol. 36. American Mathematical Society, Providence (1969)

15. Gonchar, A.A.: On convergence of Padé approximants for some classes of meromorphic functions Math. USSR Sb. 26, 555-575 (1975)

16. Gonchar, A.A.: On the convergence of generalized Padé approximants of meromorphic functions. Math. USSR Sb. 27, 503-514 (1975)

17. Gonchar, A.A., Rakhmanov, E.A., Suetin, S.P.: On the convergence of Padé approximations of orthogonal expansions. Proc. Steklov Inst. Math. 200, 149-159 (1993)

18. Gonchar, A.A., Rakhmanov, E.A., Suetin, S.P.: On the rate of convergence of Padé approximants of orthogonal expansions. In:Progress in Approximation Theory, pp. 169-190. Springer, New York (1992)

19. Gonchar, A.A.: Rational approximation of analytic functions. Proc. Steklov Inst. Math. 272, S44-S57 (2011)

20. Kaliaguine, V.A.: A note on the asymptotics of orthogonal polynomials on a complex arc: the case of a measure with a discrete part. J. Approx. Theory 80, 138-145 (1995)

21. Lubinsky, D.S., Sidi, A.: Convergence of linear and nonlinear Padé approximants from series of orthogonal polynomials. Trans. Am. Math. Soc. 278, 333-345 (1983)

22. Maehly, H.J.: Rational approximations for transcendental functions. In: Proceedings of the International Conference on Information Processing, Butterworths, pp. 57-62 (1960)

23. Miña-Díaz, E.: An asymptotic integral representation for Carleman orthogonal polynomials. Int. Math Res. Not. 2008, 1-38 (2008)

24. Miña-Díaz, E.: An expansion for polynomials orthogonal over an analytic Jordan curve. Commun. Math. Phys. 285, 1109-1128 (2009)

25. Miña-Díaz, E.: Asymptotics for polynomials orthogonal over the unit disk with respect to a positive polynomial weight. J. Math. Anal. Appl. 372, 306-315 (2010)

26. Simanek, B.: Ratio asymptotics, Hessenberg matrices, and weak asymptotic measures. Int. Math. Res. Not. 24, 6798-6825 (2014)

27. Simon, B.: Orthogonal Polynomials on the Unit Circle, vol. 54, Parts I and II. Colloquium Publications, American Mathematical Society, Providence (2005)

28. Simon, B.: Szegő's Theorem and its Descendants. Princeton University Press, Princeton (2011)

29. Sobczyk, G.: Generalized Vandermonde determinants and applications. Aportaciones Matematicas, Serie Comunicaciones 30, 203-213 (2002)

30. Stahl, H., Totik, V.: General orthogonal polynomials. In: Encyclopedia of Mathematics and its Applications, vol. 43. Cambridge University Press, Cambridge (1992)

31. Stylianopoulos, N.: Strong asymptotics for Bergman polynomials over domains with corners and applications. Constr. Approx. 38, 59-100 (2013)

32. Suetin, P.K.: Fundamental properties of polynomials orthogonal on a contour. Russ. Math. Surv. 21 , 35-83 (1966)

33. Suetin, P.K.: Polynomials Orthogonal over a Region and Bieberbach Polynomials. American Mathematical Society, Providence (1974) 
34. Suetin, S.P.: On the convergence of rational approximations to polynomial expansions in domains of meromorphy of a given function. Math. USSR Sb. 34, 367-381 (1978)

35. Suetin, S.P.: Inverse theorems on generalized Padé approximants. Math. USSR Sb. 37, 581-597 (1980)

36. Suetin, S.P.: On Montessus de Ballore's theorem for rational approximants of orthogonal expansions. Math. USSR Sb. 42, 399-411 (1982)

37. Suetin, S.P.: On an inverse problem for the $m$ th row of the Padé table. Sb. Math. 52, 231-244 (1985)

38. Suetin, S.P.: Asymptotics of the denominators of the diagonal Padé approximations of orthogonal expansions. Dokl. Ross. Akad. Nauk. 56, 774-776 (1997)

39. Suetin, S.P.: Padé approximants and efficient analytic continuation of a power series. Russ. Math. Surv. 57, 43-141 (2002)

40. Szegő, G.: Über orthogonale Polynome, die zu einer gegebenen Kurve der komplexen Ebene gehören. Math. Z. 9, 218-270 (1921)

41. Szegő, G.: Orthogonal Polynomials, vol. 23, 4th edn. Amer. Math. Soc. Colloq. Publ. American Mathematical Society, Providence (1975)

42. Widom, H.: Extremal polynomials associated with a system of curves in the complex plane. Adv. Math 3, 127-232 (1969) 